\title{
Nivel de desarrollo motor grueso en preescolares de México sin profesores de educación física
}

\author{
Level of development thick engine in preschools in Mexico \\ without professors of physical education
}

* María Bermudez Ferrales, ** Felipe Poblete Valderrama, * Antonio Pineda Espejel, * Nohemí Castro Rodriguez, *** Faubricio Inostroza Ordenes

Bermudez, M.; Poblete, F.; Pineda, A.; Castro, N. \& Inostroza, F. (2018). Nivel de desarrollo motor grueso en preescolares de México sin profesores de educación física. Revista Ciencias de la Actividad Física UCM, N 19(1) enero-junio, 75-81. DOI: http://doi.org/10.29035/rcaf.19.1.8

\section{RESUMEN}

La investigación tuvo como objetivo identificar si los niños del jardín infantil María Esther de Mexicali Baja California México, sin profesor de Educación Física tienen un desarrollo motor acorde a su edad cronológica. Se utilizó un enfoque cuantitativo, con alcance descriptivo con un diseño no experimental de corte transeccional. La muestra fue no probabilística por juicio y la conformaron un total de 36 preescolares. Estos escolares fueron sometidos al test de desarrollo motor grueso TGMD-2. A partir de los datos recogidos, se obtuvo que un 75\% (n:27) de los niños(as) evaluados se ubican en categorías de Promedio, Sobre el promedio Superior y Muy superior, siendo un 50\% (n:18) niñas y un 25\% (n:9) niños. En función a estos resultados, se puede concluir que el nivel de Desarrollo Motor Grueso es el esperado para la edad en la mayoría de los evaluados aun cuando existe la ausencia del profesor de Educación Física.

\section{PALABRAS CLAVE}

Preescolar, desarrollo motor grueso, profesor de Educación Física, motricidad.

\begin{abstract}
The research had as objective to identify if the children of kindergarten Maria Esther of Mexicali Baja California Mexico, without professor of Physical Education have a motor development according to their chronological age. We used a quantitative approach, with descriptive scope with a non-experimental design of transectional cut. The sample was non-probabilistic by trial and made up a total of 36 preschoolers. These students were submitted to the TGMD- 2 gross motor development test. From the data collected, it was obtained that $75 \%$ (n: 27) of the evaluated children are placed in categories of Average, Over average, Higher and Very superior, being 50\% (n: 18) Girls and 25\% (n: 9) children. Based on these results, it can be concluded that the level of Gross Motor Development is the expected level for the age in the majority of the evaluated ones even if there is absence of the professor of Physical Education.
\end{abstract}

\section{Key words}

Preschool, Heavy Motor Development, Physical Education Teacher, Motricity.

* Maestría en Educación Física y Deporte Escolar, Facultad de Deportes. Universidad Autónoma de Baja California, México.

** Universidad Santo Tomás, Sede Valdivia.

*** Escuela F-929 “11 de septiembre”, Departamento de Educación, Los Ángeles - Chile. 


\section{INTRODUCCIÓN}

El presente estudio estableció como objetivo, identificar si los niños del jardín infantil María Esther de Mexicali Baja California México, sin profesor de Educación Física tienen un desarrollo motor acorde a su edad cronológica, utilizando el test TGMD-2.

Por desarrollo motor se entenderá el cambio progresivo en el comportamiento motor a lo largo del ciclo de la vida, generado por una interacción entre los requerimientos de la tarea, la biología del individuo y las condiciones del ambiente (Gallahue, 2003).

El desarrollo motor adecuado se puede apreciar con la evolución de la especialización de acciones motrices que se llevan a cabo desde el nacimiento y a lo largo de la adultez, para luego iniciar una involución en la vejez. El desarrollo de las competencias motrices, pasa por una serie de cambios a lo largo de la vida, principalmente determinados por el funcionamiento del sistema perceptivo motor, que permite que los movimientos rudimentarios se conviertan en movimientos coordinados, los cuales les permiten a los seres humanos relacionarse con su mundo y los demás (Ruiz, 1994).

En los primeros años de vida se producen cambios notables en relación con el desarrollo motor, en este proceso la percepción, a través de los sentidos, tiene un papel importante; transitan de una situación de total dependencia a una progresiva autonomía; pasan del movimiento descontrolado al autocontrol de estos. Cambios que se relacionan con el proceso madurativo del cerebro se dan en cada individuo y con las experiencias que las niñas y los niños viven en los ambientes donde se desenvuelven (Guía para la Educadora, 2011).

Según la UNESCO, 2015 el currículo de la Educación Física de Calidad (EFC) promueve la competencia motriz para estructurar el pensamiento, expresar sentimientos y enriquecer la comprensión.

Luarte, Poblete y Flores (2014) manifiestan que los profesionales de la educación física, a través de ésta, permiten que diferentes aspec- tos de la vida del ser humano se integren en una red de relaciones que definen características especiales en cada persona, permitiendo que los niños puedan vivir y experimentar su propia motricidad, de esa forma cada cual será capaz de enfrentar situaciones y sensaciones, tanto a nivel individual como grupal que, trascenderán en el tiempo, ya sea como experiencias positivas o negativas, según sus capacidades o limitaciones.

Todos necesitamos movernos para lograr el desarrollo de nuestras potencialidades biológicas, psíquicas y físicas. Cada aprendizaje, cada práctica, es una nueva experiencia que viene a complementarse con todas aquellas que se van adquiriendo a lo largo de nuestro crecimiento y desarrollo como seres humanos.

En cada etapa del crecimiento, existen habilidades y capacidades motrices que vienen determinadas desde que nacemos. Poblete, Morilla y Quintana (2015) indican que estas se desarrollarán de acuerdo a la estimulación y los aprendizajes motores que se van alcanzando, a través del medio familiar y educativo en el cual se desenvuelve el niño.

En esta necesidad de conocer y aprender en contacto con la exploración del medio y del movimiento, el niño comienza a generar avances y adaptaciones de sus potencialidades físicas y mentales, estableciendo relaciones con los demás y su entorno inmediato. A medida que crece, estos cambios van adquiriendo sentido de perfectibles, es decir, tiene la capacidad de mejorarlos y perfeccionarlos de acuerdo a como se vayan ejecutando, ya que las experiencias motrices tempranas son fundamentales para lograr un mejor desarrollo de la motricidad

La motricidad también abarca las habilidades del niño para moverse y desplazarse, explorar y conocer el mundo que le rodea y experimentar con todos sus sentidos (olfato, vista, gusto y tacto) para procesar y guardar la información del entorno que le rodea. Así pues, el ámbito de la motricidad está relacionado, mayormente, con todos los movimientos que de manera coordinada realiza el niño con pequeños y grandes grupos musculares, 
los cuales, son realmente importantes porque permiten expresar la destreza adquirida en las otras áreas y constituyen la base fundamental para el desarrollo del área cognitiva y del lenguaje (Villanueva, 2014).

\section{MÉTODO}

Se llevó a cabo un estudio de enfoque cuantitativo, de alcance descriptivo que buscó establecer si existía el esperado nivel de desarrollo motor grueso de los preescolares del jardín infantil María Esther de Mexicali Baja California México, el diseño del estudio fue no experimental, de corte transversal.

En el estudio participaron un total de 36 niños y niñas de los cuales 21 fueron de sexo femenino y 15 masculino en edades de 4 y 5 años, el tipo de muestreo fue no probabilístico por conveniencia.

El estudio fue desarrollado basándose en la declaración de Helsinki para el trabajo con seres humanos, para su inclusión en el estudio, los preescolares: a) No debían presentar alteraciones motoras o funcionales, que pudiesen interferir en los resultados de los test aplicados, b) Debían pertenecer al jardín infantil María Esther de Mexicali Baja California México siendo alumno regular con una antigüedad mínima de 6 meses, c) Debían asistir el día de las evaluaciones con la indumentaria adecuada para realizar actividades deportivas, es decir, ropa cómoda y zapatillas. Todos los alumnos participantes del estudio contaban con un consentimiento informado firmado por sus apoderados y las mediciones se realizaron en el mes de noviembre del 2016.

El análisis estadístico fue de tipo descriptivo, analizando tendencias centrales y frecuencias mediante el programa Excel.

Las mediciones fueron realizadas en el gimnasio del jardín seleccionado, facilitado por las autoridades de este y utilizadas en su oportunidad de forma exclusiva para la investigación.

Se utilizó el test de desarrollo motor grueso TGMD-2 (Test of Gross Motor Development, second edition), que evalúa la coordinación entre el tronco y las extremidades durante el gesto motor de niños(as) con edades de tres años cero meses a diez años once meses. El test posee una confiabilidad de $0.91 \mathrm{y}$ una validez de constructo, contenido y predictiva moderada (Ulrich, 2000).

Cada habilidad motora gruesa incluye varios componentes de comportamiento (para habilidades Locomotoras se encuentran las subpruebas de: carrera, galopar, saltar a un pie alternado, saltar hacia delante, salto horizontal, deslizamiento; mientras que para las subpruebas de manipulación encontramos: batear bola estacionaria, drible estacionario, tomar, golpear, lanzamiento de a pelotita, hacer rodar una pelotita), que se presentan como criterios de desempeño. En general, estos comportamientos representan un patrón maduro de la habilidad. Si el niño realiza un comportamiento de componente correcto, el examinador marca un 1; si el niño realiza un componente conductual incorrecto, el examinador marca un 0 . Después de completar este procedimiento para cada uno de los dos ensayos, el examinador saca las puntuaciones totales de los dos ensayos para obtener una puntuación de habilidad para cada tema. Las pruebas fueron controladas por los investigadores del presente artículo quienes poseen dominio del Test en cuanto aplicación e interpretación de datos. 


\section{RESULTADOS}

A continuación, se presentan los resultados obtenidos tras la aplicación del Test TGMD-2.

Tabla 1

Análisis descriptivo de resultados TGMD-2 niños(as) 4 y 5 años de edad.

\begin{tabular}{lcccccccccc}
\hline \multirow{2}{*}{ Sujetos } & $\begin{array}{c}\text { Edad } \\
\text { (Años) }\end{array}$ & \multicolumn{3}{c}{ Test Locomotor } & \multicolumn{3}{c}{ Test Manipulativo } & \multicolumn{3}{c}{ Cuociente Motor } \\
\cline { 3 - 11 } & & Mínimo & Máximo & Promedio & Mínimo & Máximo & Promedio & Mínimo & Máximo \\
\hline Niño(as) & 4 & 17 & 29 & 24,87 & 14 & 25 & 20 & 88 & 109 \\
\hline Niño(as) & 5 & 18 & 46 & 32,89 & 12 & 40 & 25,39 & 70 & 154 \\
\hline
\end{tabular}

La Tabla 1. Análisis descriptivo de los resultados TGMD-2 Niños(as) 4 y 5 años de edad, permite observar que según puntajes absolutos los niños(as) de 5 años presentan mejo- res niveles de puntación en relación a los de niños(as) de 4 años en Test Locomotor, Test Manipulativo y Cuociente Motor.

Tabla 2

Clasificación General TGMD-2 niños(as) 5 años de edad.

\begin{tabular}{lcccccc}
\hline Categorías clasificación general & Niños & $\%$ & Niñas & $\%$ & Total Muestra & $\%$ \\
\hline Muy superior & 1 & 7,14 & 2 & 14,3 & 3 & 11 \\
\hline Superior & 2 & 14,3 & 1 & 7,1 & 3 & 11 \\
\hline Por encima del promedio & 0 & 0 & 2 & 14,3 & 2 & 7 \\
\hline Promedio & 5 & 35,7 & 9 & 64,3 & 3 & 50 \\
\hline Por debajo del promedio & 3 & 21,42 & 0 & 0 & 2 & 11 \\
\hline Pobre & 2 & 14,3 & 0 & 0 & 1 & 7 \\
\hline Muy pobre & 1 & 7,14 & 0 & 0 & 28 & 100 \\
\hline Total & 14 & 100 & 14 & 100 & & 3 \\
\hline
\end{tabular}

Tabla 2. Clasificación General TGMD-2 niños(as) 5 años de edad, muestra que del total de la muestra un 79\% (n:22) se encuentra en niveles esperados y superiores para su edad, siendo las niñas (n:14) las que se ubican en mejores categorías esperadas o superiores para la edad que los niños (n:8).

Tabla 3

Clasificación General TGMD-2 Niños(as) 4 años de edad.

\begin{tabular}{lcccccc}
\hline Categorías clasificación general & Niños & $\%$ & Niñas & $\%$ & Total Muestra & $\%$ \\
\hline Muy superior & 0 & 0 & 0 & 0 & 0 & 0 \\
\hline Superior & 0 & 0 & 0 & 0 & 0 & 0 \\
\hline Por encima del promedio & 0 & 0 & 0 & 0 & 0 & 0 \\
\hline Promedio & 1 & 100 & 4 & 57.15 & 5 & 62.5 \\
\hline Por debajo del promedio & 0 & 0 & 3 & 42.85 & 3 & 37.5 \\
\hline Pobre & 0 & 0 & 0 & 0 & 0 & 0 \\
\hline Muy pobre & 0 & 0 & 0 & 0 & 0 & 0 \\
\hline Total & 1 & 100 & 7 & 100 & 8 & 100 \\
\hline
\end{tabular}


Tabla 3. Clasificación General TGMD-2 niños(as) 4 años de edad, expone que la muestra en este nivel mayoritariamente esta compuesta por niñas $87,5 \%(\mathrm{n}: 7)$, muestran que en relación a los niveles de categorización de desarrollo motor de niños un 100\%(n:1) se ubican en categoría promedio para la edad y un $42.85 \%$ (n:3) de las niñas se categorizan en niveles bajos o no esperados para la edad.

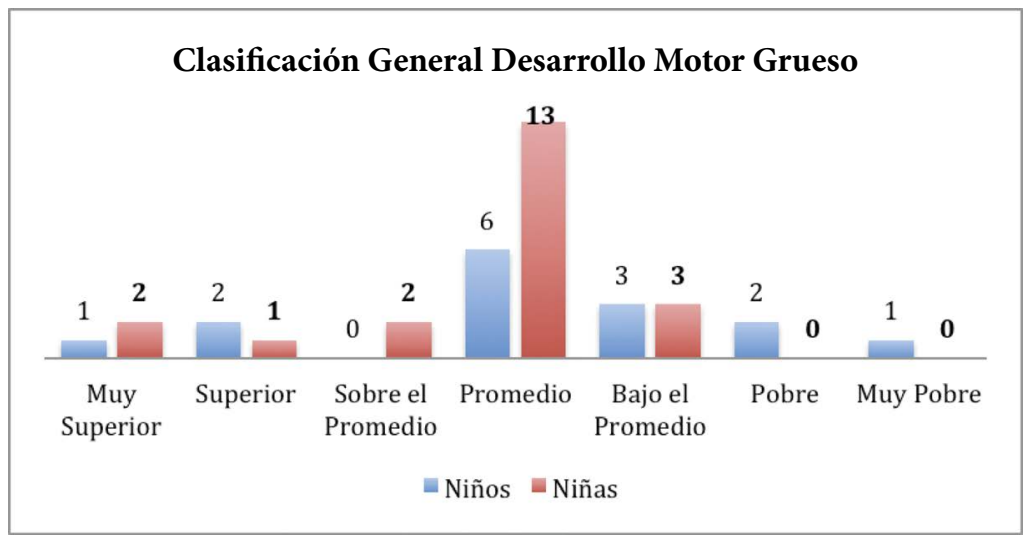

Figura 1. Clasificación General Desarrollo Motor Grueso niños(as) 4 y 5 años de edad.

La figura 1 Muestra que un 75\% (n:27) de los niños(as) evaluados se ubican en categorías esperadas o superiores de las esperadas para su edad, siendo un $50 \%$ (n:18) niñas y un $25 \%$ (n:9) niños.

\section{DISCUSIÓN}

En relación a los datos obtenidos se puede apreciar que los preescolares Mexicanos evaluados sin la presencia de un profesor de Educación Física, presentan en un $75 \%$ categorias esperadas o superiores para su edad cronológica lo que se contradice por lo pesquizado en tres estudios Chilenos realizado por Luarte, Poblete y Flores (2014) quienes presentan un $93,1 \%$ categorias esperadas e inferiores para la edad y el estudio de Poblete, Morilla y Quintana (2015) en donde un total de 60,4\% se categorizan en niveles bajos para la edad en preescolares sin intervencion de profesores de Educación Física y finalmente la investigación de Luarte, Flores y Poblete (2012) quienes tambien concluyeron que el nivel de desarrollo motor grueso de escolares de NB2 con presencia de profesores de Educación Física se encuentran en categorias bajas para la edad, lo que evidencia que existen distintas realidades en cuanto al desarrollo motor entre paises Latinoamericanos que tienen ausencia o presencia de profesor de Educación Física en la primera infancia.

Al observar los hallazgos del presente estudio realizado en preescolares Mexicanos urbanos coincide con los investigado por Luna y Poblete (2011) quienes exponen que escolares de NB1 de zona rural presentaron niveles de desarrollo motor grueso esperados para la edad sin la intervención de profesionales de Educación Física.

A su vez los resultados de Poblete y cols. (2016) nos dejan en evidencia que escolares de 6 a 8 años de edad en un estudio comparativo de zona urbana y rural se categorizan en un $93,7 \%$ y $94 \%$ respectivamente en categorias esperadas y superiores a su edad cronológica, 
lo que deja en evidencia que la caracterización geográfica no es un factor que norma buenos o malos niveles de desarrollo motor grueso.

La importancia de una estimulación motriz adecuada liderada por profesionales de Pedagogía en Educación Física permitirá mejorar o mantener los buenos resultados obtenidos en el presente estudio, lo que se relaciona con lo planteado por la OMS (2009) en donde indica que la estimulación motriz en los primeros años de vida (período prenatal hasta los ocho años) es relevante para su desarrollo a nivel mental, emocional y físico. En esta misma linea Campos (2010), corrobora la importancia de un desarrollo motor acorde a la edad del niño, demostrando que este influye en el aspecto cognitivo, donde el desarrollo motor esperado para su edad presentará igualmente un desarrollo cognitivo y de lenguaje acorde, por lo que la estimulación para la mantención o mejora de los niveles de desarrollo motor favoreceran el desarrollo integral del niño(a).

\section{CONCLUSIÓN}

Una vez expuesto los resultados se puede concluir que, los niños evaluados mostraron que un 75\% (n:27) se ubican en promedio o por encima de él, por lo tanto, se determina que se categorizan en una edad motora acorde con su edad cronológica según el Test TGMD-2.

A pesar de no existir la presencia del profesor de Educación Física, estos niños han logrado obtener un resultado favorable, por lo que se supone que tanto el entorno como la biología de estos niños ha logrado favorecer ampliamente al Desarrollo Motor Grueso, variables que sería interesante que futuros estudios las puedan abordar y relacionar.

Es importante rescatar la importancia de la presencia del profesor de Educación Física en estas edades para la estimulación de un desarrollo Motor Grueso acorde a la edad, tomando en cuenta la nueva propuesta educativa que propone la secretaria de educación y cultura de México, que intenta establecer en sus programas la importancia de la motricidad gruesa en todas las edades de educación básica preescolar, primaria y secundaria.

\section{REFERENCIAS BIBLIOGRÁFICAS}

Campos, L. (2010). Importancia del desarrollo motor grueso en relación a los procesos evolutivos del lenguaje y la cognición en niños de 3 a 7 años de la ciudad de Barranquilla. Revista Salud Uniforme. Vol. 26(1). 3.

Gallahue, D. (2003). Developmental Physical Education for All Children. 4th Edition. Human Kinetics. Auckland.

Guía para la educación pública (2011). Guía para la educadora. Educación Básica y Preescolar. Dirección General de Desarrollo Curricular y Dirección General de Educación continua. México: Secretaría de Educación Pública.

Luarte, C. Flores, C. Poblete, F. (2012) Nivel de Desarrollo Motor Grueso en escolares de NB2 de Talcahuano, Octava RegiónChile. Revista Ciencias de la Actividad Física UCM. Vol. 13 51-57.

Luarte, C. Poblete, F. Flores, C. (2014). Nivel de desarrollo motor grueso en preescolares sin intervención de profesores de Educación Física, Concepción, Chile. Revista Ciencias de la Actividad Física UCM. 15(1) 7-16.

Luna, P. \& Poblete, F. (2011). Desarrollo motor grueso en escolares sin intervención de profesionales de Educación Física del NB1 del Microcentro "Amanecer" de la comuna de Nacimiento (Región del BIOBÍO, Chile). Revista Horizonte Ciencias de la Actividad Física, 2(2) 2535.

Organización Mundial de la Salud. (2009). Desarrollo en la primera infancia. Centro de prensa. Nota descriptiva No332.

Poblete, F. Morilla, C. Quintana, C. (2015). Nivel de desarrollo motor grueso en pre-escolares sin intervención de profesores de Educación Física, Valdivia. Revista Horizonte Ciencias de la Actividad Física, 6(2) 33-40. 
Poblete, F. Guerra, R. Toro, P. Cruzat, E. (2016).

Desarrollo motor grueso en escolares de zona Urbana y Rural. Revista Horizonte Ciencias de la Actividad Física, 7(1) 59-66.

Programa de estudio 2011 guía para la educadora. (2011). Mexico: Secretaría de Educación y Cultura, pp.68-72.

Ruiz, L. M. (1994). Deporte y aprendizaje: Procesos de adquisición y desarrollo de habilidades. Madrid: Antonio Machado.

Ulrich, D. (2000). Test of Gross Motor Devolepment. (2a Ed.). Austin: Proed; 2000. pp.1-60

UNESCO (2015). Educación Física de Calidad. París: UNESCO, p.14.

Villanueva, M. (2014). Desarrollo Motor en los Niños y Niñas de 2 Años de las Salas de Estimulación Temprana en el Distrito de Chimbote en el Año 2014. Segunda Especialidad en Educación Básica Especial con Mención en Educación y Aprendizajes Tempranos. Universidad Católica los Ángeles Chimbote.

\section{Dirección para correspondencia}

Felipe Poblete Valderrama

Licenciado en Educación.

Profesor de Educación Física.

Magíster en Educación en Salud y Bienestar

Humano.

Dr @ en Educación.

Coordinador Académico Postgrados e

Investigación.

Universidad Santo Tomás, Sede Valdivia,

Chile.

Contacto:

felipepobletev@gmail.com

Recibido: $31 / 08 / 2017$

Aceptado: 31/05/2018 